УДК 621.373:535

\title{
Amplification of Light by Molecules with Selectively Oriented States in the Pulsed Electric Field
}

\author{
Igor A. Kuzovatov* \\ Institute of Mathematics and Computer Science \\ Siberian Federal University \\ Svobodny, 79, Krasnoyarsk, 660041 \\ Russia \\ Vitaliy V. Slabko ${ }^{\dagger}$ \\ Alexey V. Shamshurin ${ }^{\ddagger}$ \\ Institute of Engeneering Physics and Radio Electronics \\ Siberian Federal University \\ Svobodny, 79, Krasnoyarsk, 660041
}

Russia

Received 30.01.2016, received in revised form 16.03.2016, accepted 19.05.2016

The possibility of inversionless amplification of light by dichroic molecules that can be oriented selectively over states by application of an pulsed electric field is analyzed. The numerical simulation of the orientation process of molecules in the external field is considered. As shown the conditions of inversionless amplification with considering of transition between energetic levels may be implemented.

Keywords: inversionless light amplification, molecule, exponential fitting.

DOI: 10.17516/1997-1397-2016-9-3-332-337.

\section{Introduction}

When analyzing light interaction that is resonant to energy-level transition, the equality of probabilities of induced emitted and absorbed radiation is expected. According to quantum gain of light it requires to create the inversion of population of energy levels. However, the power of induced emitted and absorbed radiation is determined not only by the population of energy levels of particles, but also by the probabilities of induced transitions, as well as the number of energy-degenerate states. Therefore, population inversion is generally a particular case in which the emission power exceeds the absorption power and, hence, amplification of light takes place [1].

The possibility of inversionless amplification of light in a system of dichroic molecules selectively oriented over states in an external field was considered in $[2,3]$. These ideas were subsequently developed by the authors in $[4,5]$ in which on the basis of the coupled system of kinetic Boltzmann equations for the ground and excited states the effect of the relation between $\tau_{2}^{e f f}$ and $\tau_{0}$ on gain $\alpha$ is numerically analyzed. The authors have shown that to achieve the inversionless amplification the value of alignment electric field $\vec{E}_{0}$ must be close to or above

\footnotetext{
*kuzovatov@yandex.ru

$\dagger$ vslabko49@mail.ru

†shamshurin.alex@mail.ru

(c) Siberian Federal University. All rights reserved
} 
the breakdown threshold of existing environments, where the studied molecules can be placed. However, if the impulse duration of $\vec{E}_{0}$ is in $10^{-9}$ sec, the electric strength of technically pure liquid increases in some times against the constant field [6].

In this paper we present the usage of the pulsed field $\vec{E}_{0}$ as orienting. To calculate the amplification factor $\alpha$ the numerical analysis of dipole molecule orientation on the basis of solutions of nonsteady kinetic equation is used.

\section{Physical Model}

When external field $\vec{E}_{0}$ is acting on a molecular medium, the molecules are oriented in the direction corresponding to the minimum of the potential energy of their interaction with this field $[7,8]$. The degree of orientation of the medium as a whole is determined by the orientation parameter $p_{j}$, presented as the interaction of the molecule with field to the energy $k T$ of thermal motion, which produces a disorienting effect. For axially symmetric molecules placed in a constant electric field $\vec{E}_{0}$, in a state of $j=1,2$ and have constant dipole moment in this state $\vec{\mu}_{j}$, the orientation parameter $p_{j}$, takes the form,

$$
p_{j}=\frac{\vec{\mu}_{j} \vec{E}_{0}}{k T}
$$

Interaction energy and, hence, the orientation degree of the molecules in the ground and excited states can be different. In this case, the probabilities of induced transitions with absorption and amplification of polarized light, which are determined by the orientation of molecules relative to the electric field direction in the probe wave, can also be different. This makes it possible in principle to create the conditions for amplification of light with a certain polarization in the absence of population inversion by controlling the degree of orientation in the ground and excited states.

The amplificatin factor $\alpha>0$ (absorption factor $\alpha<0$ ) for molecules oriented in external field $\vec{E}_{0}$ and in field $\vec{E}$ of plane-polarized probe radiation has the form

$$
\alpha=N \sigma_{0} \int_{\Omega}\left[n_{2} f_{2}\left(\Omega, E_{0}\right)-n_{1} f_{1}\left(\Omega, E_{0}\right)\right] \cos ^{2} \vartheta d \Omega
$$

here $\sigma_{0} \sim d_{21} \cos \vartheta$ is the absorption-emission cross section of a molecule, $d_{21}$ is the electric dipole moment of the transition, $\vartheta$ is the angle between the direction of the electric dipole moment of the transition $d_{21}$ and the probe electric field vector $\vec{E}, N$ is the concentration of particles, $n_{1}$ and $n_{2}$ are the relative populations of the ground and the upper states, produced by an external pump source, $n_{1}+n_{2}=1, d \Omega=\sin \vartheta d \vartheta d \phi$ is the solid angle element, $f_{1}\left(\Omega, E_{0}\right)$ и $f_{2}\left(\Omega, E_{0}\right)$ are the statistical distribution functions for orientations of molecules in the ground and upper states, which are normalized to unity.

Let us suppose that molecules with energy levels $W_{1}$ and $W_{2}$ are in thermodynamic equilibrium with the pump field ensuring relative populations $n_{1}$ and $n_{2}$ of the levels, respectively, such that $W_{1}<W_{2}$. In this case, $n_{1}>n_{2}$, population inversion does not take place, and lifetimes $\tau_{1}^{e f f}$ and $\tau_{2}^{e f f}$ of the ground and upper states are being defined as

$$
\frac{1}{\tau_{1}^{\text {eff }}}=w_{12}^{\text {ind }}, \quad \frac{1}{\tau_{2}^{e f f}}=w_{21}^{\text {ind }}+w_{21}^{s p}, \quad \frac{\tau_{2}^{\text {eff }}}{\tau_{1}^{\text {eff }}}=\frac{n_{2}}{n_{1}},
$$

here $w_{12}^{\text {ind }}=w_{21}^{\text {ind }}$ is the probability of induced transitions and $w_{21}^{s p}$ is the probability of spontaneous transitions. 
The coupled nonstationary system of equations for functions $f_{1}\left(\Omega, E_{0}\right)$ and $f_{2}\left(\Omega, E_{0}\right)$ with the boundary (5) and initial (6) conditions and the normalization condition (7) combined with relation (2), makes it possible to solve the problem of calculation of the amplification factor formulated above, [8],

$$
\left\{\begin{array}{c}
\frac{W}{k T} \frac{\partial f_{1}}{\partial t}-\frac{1}{\sin \vartheta} \frac{\partial}{\partial \vartheta}\left(\sin \vartheta\left(\frac{\partial f_{1}}{\partial \vartheta}+f_{1} \frac{\partial}{\partial \vartheta}\left(\frac{\mu_{1} E_{0}}{k T}\right)\right)\right)=-\frac{W}{k T} w_{12}^{i n d} f_{1}+\frac{W}{k T}\left(w_{21}^{i n d}+w_{21}^{s p}\right) f_{2} \\
\frac{W}{k T} \frac{\partial f_{2}}{\partial t}-\frac{1}{\sin \vartheta} \frac{\partial}{\partial \vartheta}\left(\sin \vartheta\left(\frac{\partial f_{2}}{\partial \vartheta}+f_{2} \frac{\partial}{\partial \vartheta}\left(\frac{\mu_{2} E_{0}}{k T}\right)\right)\right)=-\frac{W}{k T}\left(w_{21}^{i n d}+w_{21}^{s p}\right) f_{2}+\frac{W}{k T} w_{12}^{i n d} f_{1} \\
\left.\frac{\partial f_{1}}{\partial \vartheta}\right|_{\vartheta=0}=\left.\frac{\partial f_{2}}{\partial \vartheta}\right|_{\vartheta=0}=0,\left.\quad \frac{\partial f_{1}}{\partial \vartheta}\right|_{\vartheta=\pi}=\left.\frac{\partial f_{2}}{\partial \vartheta}\right|_{\vartheta=\pi}=0 \\
f_{1}(0, \vartheta)=g_{1}(\vartheta), \quad f_{2}(0, \vartheta)=g_{2}(\vartheta), \\
2 \pi \int_{0}^{\pi}\left(g_{1}(\vartheta)+g_{2}(\vartheta)\right) \sin \vartheta d \vartheta=1 .
\end{array}\right.
$$

Here $W=6 k T \tau_{0}$ is the responsible for the scale model parameter, $\tau_{0}$ is the time of stabilization of orientational equilibrium. For numerically solving this problem the exponentional fitting method is used [9].

\section{Computing experiment}

Let us consider the two following cases, in which the effect of inversionless amplification can be manifested most strongly [2-5]:

1. Let us suppose that the constant dipole moment $\vec{\mu}_{1}$ in the ground state is smaller than dipole moment $\vec{\mu}_{2}$ in the excited state. In this case the effect of inversionless amplification can be manifested in parallel fields $\vec{E}$ and $\vec{E}_{0}$, then $\vartheta=\vartheta_{0}$ and the amplificatin factor $\alpha,(2)$, is given by the formula

$$
\alpha=\sigma_{0} \int_{0}^{2 \pi} \int_{0}^{\pi}\left(n_{2} f_{2}(\vartheta)-n_{1} f_{1}(\vartheta)\right) \cos ^{2} \vartheta d \vartheta d \varphi .
$$

2. If the opposite condition $\left|\vec{\mu}_{2}\right|<\left|\vec{\mu}_{1}\right|$ is satisfied, then the molecules in the ground state are predominantly oriented along field $\vec{E}_{0}$. Therefore, to suppress absorption, it is expedient to orient field $\vec{E}_{0}$ orthogonally to field $\vec{E}$. The amplificatin factor $\alpha$ averaged over molecular orientations has the form

$$
\alpha=\sigma_{0} \int_{0}^{2 \pi} \int_{0}^{\pi}\left(n_{2} f_{2}(\vartheta)-n_{1} f_{1}(\vartheta)\right) \sin ^{3} \vartheta d \vartheta \cos ^{2} \varphi d \varphi .
$$

Let us consider the influence of the orientation parameters $p_{1}$ and $p_{2}$ on gain $\alpha$. The problem (4)-(7) can be solved numerically by the exponential fitting method under the following physical parameters: $\tau_{0} / \tau_{1}^{e f f}=0.01, \tau_{0} / \tau_{2}^{e f f}=0.0125$, that corresponding to ratio populations of the states $n_{2} / n_{1}=0.8$. The external electric field pulse duration $1.0 \cdot 10^{-9} \mathrm{sec}$ assumed to be equal to $E_{0}=2 M V / s m$, that two times above the breakdown threshold of existing environments. The time $\tau_{0}$ of stabilization of orientational equilibrium assumed to be equal to $\tau_{0}=0.25 \cdot 10^{-9} \mathrm{sec}$

Fig. $1 a$ and $1 b$ show the calculated dependences of the amplificatin factor $\alpha$ as a function of the orientation parameters. Curve (1) corresponds to the situation in which the external electric 
field $E_{0}=1 \mathrm{MV} / \mathrm{sm}$ and curve (2) corresponds to the situation in which the external electric field pulse $E_{0}=2 \mathrm{MV} / \mathrm{sm}$. The orientation parameters of the assumed field $E_{0}$ are the same value as the dipole moments $\mu_{1}$ and $\mu_{2}$, that are used in papers $[4,5,9]$.

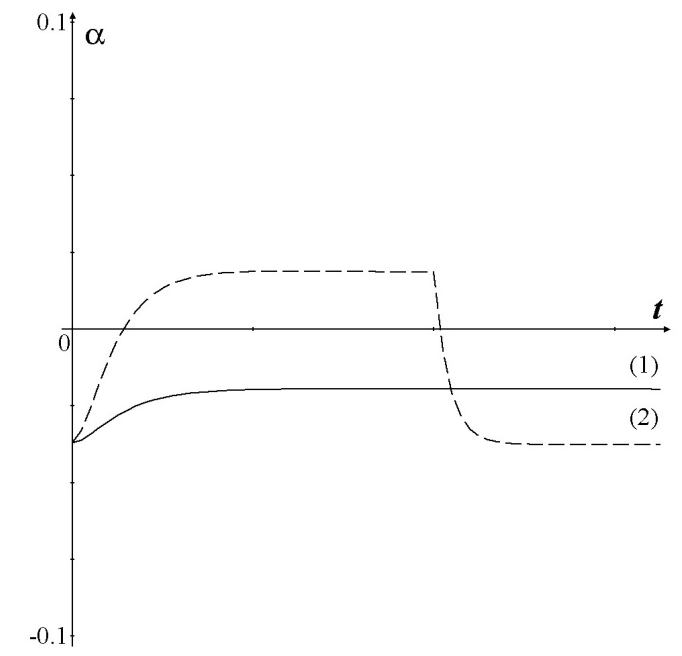

a) $\left|\vec{\mu}_{1}\right|<\left|\vec{\mu}_{2}\right|$,

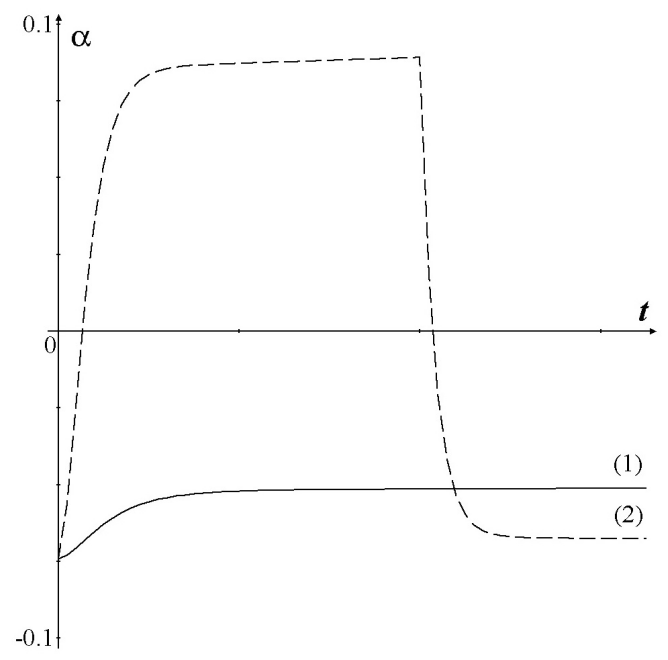

b) $\left|\vec{\mu}_{1}\right|>\left|\vec{\mu}_{2}\right|$,

(1) $p_{1}=1, p_{2}=0.25$, (2) $p_{1}=2, p_{2}=0.5$

Fig. 1. The influence of the orientation parameters $p_{1}$ and $p_{2}$ on gain $\alpha$

Note that, in the first case, $p_{2} / p_{1}=4$ and external field is a static the parameter $\alpha$ is negative (absorption) and its maximum value is $\alpha_{\max }=-0.019$. When the external field is pulse the parameter $\alpha$ is positive (amplification) and its maximum value is $\alpha_{\max }=0.019$. In the second case when $p_{1} / p_{2}=4$ and external field is a static the parameter $\alpha$ is negative (absorption) and its maximum value is $\alpha_{\max }=-0.051$. The positive magnitude of amplification factor $\alpha$ can be reached by switching the external field in the pulsed regime, $\alpha_{\max }=0.089$.

Let us analyze a character of amplification factor modifications. The maximum amplification factor is observed in the case $\mu_{1}>\mu_{2}$, (Fig. 1b). Let us consider a limit situation: $\mu_{1}>\mu_{2}$, $\mu_{2}=0$. In this case, particles in a ground state are oriented lengthwise the $\vec{E}_{0}$ field, the orientation of the particles in upper state is isotropic. If the vectors $\vec{E}$ and $\vec{E}_{0}$ are orthogonally directed, the influence of the particles in ground state does not decrease the amplification field $\vec{E}$ and the amplification factor $\alpha$ is determined by the first term of (9).

Let us consider the reverse situation: $\mu_{2}>\mu_{1}, \mu_{1}=0$. In this case the particles in upper state are oriented lengthwise the $\vec{E}_{0}$ field and in their ground state the orientation of the particles is isotopic. The amplification factor $\alpha$ is determined by the gain of the particles in upper state as well as the absorption of particles in ground state, (8).

In general case if $\mu_{1}$ and $\mu_{2}$ are different from 0 , when meeting the condition $\mu_{1}>\mu_{2}$, the greater degree of orientation is reflected on the particles in ground state, and when the orientation of the vectors of the strengthened field $\vec{E}$ is orthogonal to $\vec{E}_{0}$. 


\section{Conclusion}

The case study of the investigated sphere is organic molecules, dye molecules in particular. The lifetime in upper state for electric dipole transition of the following molecules is equal to the size of order $10^{-8}-10^{-9} \mathrm{sec}$. Orientation time may vary widely depending on the different parameters, that is medium viscosity and the size of molecules, and may also fall within the limits of $10^{-10}-10^{-12} \mathrm{sec},[7,8,10]$. The constant dipole moment corresponds to the value of some Debyes. The usage of pulsed orient field produces parameter values of orientation greater then unity in the pre-breakdown field.

Numerical analysis shows that when the parameters of sphere and external field are real, the inversionless gain may be reached on condition of molecule orientation in the pulsed field. The following findings provide the basis for full-scale experiments.

This research is partially supported by Grant of the Ministry of Education and Science of the Russian Federation for Siberian Federal University (projects 3.1749.2014/K, 214/71) and RFBR, research project 14-02-00219-a.

\section{References}

[1] J.Mompart, R.Corbalan, Lasing without inversion, Journal of Optics B: Quantum and Semiclassical Optics, 3(2000), no. 2, R7-R24.

[2] A.K.Popov, V.V.Slabko, Switching from opaque via transparent to a strongly amplifying state is shown to be possible for molecular media without a change in the noninverted population of their energy levels, Optics Letters, 30(2005), no. 13, 1719-1721.

[3] A.K.Popov, V.V.Slabko, Optical switching and inversionless amplification controlled by statedependent alignment of molecules, Journal of Physics B: Atomic, Molecular and Optical Physics, 38(2005), no. 11, 1787-1796.

[4] I.A.Kuzovatov, V.V.Laschinsky, V.V.Slabko, A.V.Shamshurin, Influence of the orientational equilibrium time on the inversionless light amplification effect with selective on the states of oriented molecules, Journal of Siberian Federal University, Mathematics \& Physics, 4(2011), no. 2, 240-248 (in Russian).

[5] V.V.Slabko, I.A.Kuzovatov, A.V.Shamshurin, On the possibility of inversion-free amplification of light by dichroic molekules in an external orienting field, Technical Physics, $\mathbf{5 7}(2012)$, no. 2, 214-219.

[6] G.A.Mesyats, Pulse Power Engineering and Electronics, Plenum, New York, 2004.

[7] N.G.Bakhshiev, Spectroscopy of Intermolecular Interactions, Leningrad, Nauka, 1972 (in Russian).

[8] S.Kelikh, Molecular Nonlinear Optics, Moscow, Nauka, 1981 (in Russian).

[9] I.A.Kuzovatov, A.V.Shamshurin, Numerical modeling of the process of orientation of twolevel molecules in an external field using the method of exponential fitting, Sibirskii Zhurnal Industrial'noi Matematiki, 15(2012), no. 3, 45-57 (in Russian). 
[10] A.N.Tetenin, Photonics of dye molecules and related organic compounds, Leningrad, Nauka, 1967 (in Russian).

\section{Усиление света селективно по состояниям ориентирован- ными молекулами в импульсном электрическом поле}

Игорь А. Кузоватов Институт математики и фундаментальной информатики

Сибирский федеральный университет Свободный, 79, Красноярск, 660041

$$
\text { Россия }
$$

Виталий В. Слабко Алексей В. Шамшурин

Институт инженерной физики и радиоэлектроники

Сибирский федеральный университет Свободный, 79, Красноярск, 660041

Россия

$\overline{\text { Анализируется возможность безынверсного усиления света дихроичными молекулами, которые }}$ могут быть ориентированы селективно по состояниям путем наложения импульсного электрического поля, что позволяет значительно повысить порог пробол среды. Проведено численное моделирование прочесса ориентации молекул во внешнем поле с учетом переходов между энергетическими уровнями и показано, что условия безынверсного усиления могут быть реализованы.

Ключевые слова: безынверсное усиление света, молекула, экспоненииалъная подгонка. 